\title{
Banach spacetime-like Dvoretzky volume concentration as cosmic holographic dark energy
}

\author{
Mohamed S. El Naschie \\ Dept. of Physics, University of Alexandria, Alexandria, Egypt
}

Email address:

Chaossf@aol.com

\section{To cite this article:}

Mohamed S. El Naschie. Banach Spacetime-Like Dvoretzky Volume Concentration as Cosmic Holographic Dark Energy. International Journal of High Energy Physics. Vol. 2, No. 1, 2015, pp. 13-21. doi: 10.11648/j.ijhep.20150201.12

\begin{abstract}
We start from Banach spaces motivated theory of convex geometry in high dimensionality and give a new additional confirmation of previous derivations of the 96 per cent dark energy density of the cosmos. The result derives directly from the purely geometric-topological phenomenon of measure-mass concentration and gives an unqualified complete confirmation of our previous analysis including the hidden quantum nature of Einstein's celebrated equation $\mathrm{E}=\mathrm{mc}^{2}$. The straight forward relation to holographic dark energy and its endophysical nature is also outlined.
\end{abstract}

Keywords: Dvoretzky’s Theorem, Banach Spaces, K-Theory, Relativity, Cantorian Spacetime, Endophysics, Holographic Dark Energy

\section{Introduction}

The main message of the present paper is that dark energy is essentially geometrical-topological in origin and that WMAP and type 1a supernova are the first appropriate experiments to test Einstein's special relativity beyond any doubt. Einstein's most famous formula states the equivalence of matter and energy and relates them via the constant speed of light in the jewel of special relativity $E=\mathrm{mc}^{2}$ where $E$ is the energy, $\mathrm{m}$ is the mass and $c$ is the speed of light[1-5]. Intensive effort motivated by the unexpected discovery of the accelerated expansion of the cosmos and the related problem of the dark matter and dark energy $95.5 \%$ density deficit revealed that Einstein's energy 'density' equation is the sum of two parts [4-15]. The first is a measurable ordinary energy $\mathrm{E}(\mathrm{O})=$ $\mathrm{mc}^{2} / 22$ and the second which cannot be measured in any conventional way is the dark energy density $\mathrm{E}(\mathrm{D})=\mathrm{mc}^{2}(21 / 22)$ so that at the end $\mathrm{E}=\mathrm{E}(\mathrm{O})+\mathrm{E}(\mathrm{D})=\left(\mathrm{mc}^{2} / 22\right)+\mathrm{mc}^{2}(21 / 22)=$ $\mathrm{mc}^{2}$ [11-16]. To arrive at this result two main groups of methods were employed [5-56]. The first may be loosely described as mainly physical, based on the structure of the standard model of elementary high energy particles [10,57] while the second may be equally loosely described as largely mathematical starting from Cantor transfinite set theory [8,32].

In the present paper by contrast we first tackle the problem afresh using a purely topological-geometrical methodology based on some remarkable mathematical results from the modern theory of high dimensional convex geometry and the associated phenomenon of concentration of measure studied initially in Banach spaces and then lead to Dvoretzky's wonderful theorem and the subsequent relevant research [58-61] then contrast this novel purely mathematical derivation with our own E-infinity analysis [50-56]. In fact the present geometrical explanation of dark energy is essentially a substantiation of 'tHooft-Susskind holographic dark energy $[5,6,10]$.

We stress again that the final result is a complete confirmation of the dissection of $\mathrm{E}=\mathrm{mc}^{2}$ into $\mathrm{E}(\mathrm{O})$ of the quantum particle and $\mathrm{E}(\mathrm{D})$ of the quantum wave in addition to being a deep insight into the relation between physics, geometry and algebra as well as the unity of classical and quantum mechanics [5-78]. Finally we reason that $\mathrm{E}=\mathrm{mc}^{2}$ could be tested only via an endophysical experiment such as the cosmological measurement and the observations leading to $\mathrm{E}(\mathrm{O})=\mathrm{mc}^{2} / 22[79,80]$.

The present work is not intended to be a survey or an introduction to the problems connected to dark energy. For that purpose there is a vast volume of excellent literature for beginners as well as advanced researchers [84-89]. In particular we will not define the gravitational effect of dark energy because for us it is self evident and shown experimentally by WMAP and type 1a supernova [89]. 


\section{Background Information and Preliminary Considerations}

Cantorian spacetime is a monadic space [63] in a sense akin to that of K-theory, E-infinity rings and n-category theory [4-7]. The building blocks of this space are zero sets of a Hausdorff dimension $\phi$ embedded into empty sets of a Hausdorff $\phi^{2}$ which are distributed in a uniform randomness to form via an infinite number of unions and intersections a hierarchical-infinite dimensional Cantor set [68-77]. This Suslin-like operation [71] entails few fundamental equations which determine all what is required to deduce various vital invariant properties of this space which we use to mimic our own physical macro and micro quantum spacetime. Based on the above we were able to show in the last four years beyond reasonable doubt that the jewel of special relativity, i.e. $\mathrm{E}=\mathrm{mc}^{2}$ is in fact the sum of two partial equations. As mentioned earlier on in the introduction, the first part $\mathrm{E}(\mathrm{O})=\mathrm{mc}^{2} / 22$ gives us the real measurable energy density which is due to the particle side of quantum mechanics as modelled by the zero set in Kaluza-Klein five dimensional spacetime [30]. The second part on the other hand is the dark energy which cannot be measured in the ordinary way $\mathrm{E}(\mathrm{D})=$ $\mathrm{mc}^{2}(21 / 22)$ and which is due to the wave nature of the quantum particle-wave duality as modelled by the empty set in the same $\mathrm{K}-\mathrm{K}$ spacetime [30]. A trivial computation shows then that $1 / 22=$ $4.5 \%$ and $21 / 22=95.5 \%$ of Einstein's total energy density in complete agreement with all modern cosmological measurements. Again in the last four years numerous derivations of the preceding dissection of Einstein's basic energy-mass relation was given by the author and his collaborators using a variety of physical and algebraic derivation and all leading to the same fundamental conclusion [4-77].

The present work is not intended to review or discuss in details past derivations but is mainly concerned with giving a new purely geometrical derivation showing that the so called missing $95.5 \%$ dark energy is a well established geometrical phenomena completely understood since many years in the mathematical literature without drawing or suspecting any physical conclusion or relation to high energy quantum physics or cosmology [58-61]. We are talking here about the phenomenon of measure concentration of convex geometry with high dimensionality which is an off spring of research works on Banach spaces [66]. The next section is mainly concerned with outlining and explaining this fascinating new connection between pure geometry, quantum physics and cosmology as well as how classical mechanics arise from the deeper mechanics of the quantum and the even deeper mathematics of transfinite set theory and infinite dimensional Banach spaces [58-64].

\section{Cantorian Spacetime as a Convex Geometry in Very High Dimensions}

\subsection{Short Review of the Relevant Results in Convex Geometry}

The aim of this minimalistic section is to show the failure of our low dimensionality intuition when dealing with geometrical objects of higher dimensionality which start in earnest with $n \geq 4$ [72-74]. Similar ideas were discussed by the present author many years ago while attempting to connect string theory and the geometry of spacetime with sphere backing and chess board tiling [74]. The present fundamental example goes in the same main direction and even beyond to the degree that it becomes of fundamental importance in the cosmology of dark energy. To start let us calculate the $n-1$ dimensional slice of a unit ball. The relatively elementary consideration involving the use of the stirling formula leads to an accurate approximation of the volume of the smaller slice [58-61]

$$
\operatorname{Vol}(n)=(\sqrt{e})\left(e^{-\pi e x^{2}}\right)
$$

where $\left\{x \varepsilon R^{n}: \frac{1}{2} \leq x_{1} \leq \frac{1}{2}\right\}$ fixes the width of parallel slices. That way we can conclude that the volume 'mass' distribution is Gaussian and for a fixed slab width almost $95.5 \%$ of the mass lies in this slice [58]. Now as hinted at earlier, our intuition could lead us to wrongly think that this $95.5 \%$ measure concentration is located in dimension $n-1$ at the centre of the n-dimensional space. The mathematical-geometrical truth however is exactly the opposite and careful consideration reveals that for large $n$ the measure concentration is asymptotically near to the surface [58]. For $n=\infty$ the $95.5 \%$ is for all practical reasons the surface itself. The mathematical literature is in the meantime abounds with various extensions of this remarkable result which is based on A. Dvoretzky's theorem and the work of V. Milman and its mathematical-geometrical ramifications [58-61]. In the present work however our evident interest is clearly in the obvious relevance of the above conclusion to dark energy cosmology. We start by showing the almost one to one correspondence of the above with the Cantorian-fractal holographic boundary of E-infinity spacetime and its dark energy content.

\subsection{The Measure Concentration of E-Infinity Spacetime as an Explanation for Dark Energy}

As mentioned earlier on in the introduction, the building blocks of E-infinity Cantorian spacetime are zero sets embedded into an empty set as their surface or cobordism. Since the zero set $(\mathrm{n}=0)$ has a Hausdorff dimension $\phi$ and the empty set $(n=-1)$ has a Hausdorff dimension $\phi^{5}$ then in $\mathrm{D}=5$ Kaluza-Klein spacetime the unit volumetric measure would be the average of intersectional $\mathrm{D}=5$ zero set volume $\phi^{5}$ and the additive $5=\mathrm{D}$ empty set volume $5 \phi^{2}$ where we interpreted $\phi^{5}$ and $5 \phi^{2}$ as a Hausdorff volume or topological probability as an extension of the classical notion of geometrical probability [5-7, 68,69]. Consequently the average volume is simply [70]

$$
\langle V\rangle=\left(\phi^{5} / 2\right)+\left(5 \phi^{2} / 2\right) \text {. }
$$


where $\phi^{5}$ is Hardy's quantum entanglement [5-10]. The corresponding average energy is therefore nothing else but Einstein's celebrated formula [28]

$$
\begin{aligned}
& E=\left(\phi^{5} / 2\right) m c^{2}+\left(5 \phi^{2} / 2\right) m c^{2} \\
& =\left[\left(\phi^{5} / 2\right)+\left(5 \phi^{2} / 2\right)\right] m c^{2} \\
& =(1)\left(m c^{2}\right) \\
& =m c^{2} .
\end{aligned}
$$

From [28]

$$
\begin{aligned}
& \phi^{5} / 2=1 /(22+k) \\
& =1 /(22.18033979) \\
& \simeq 1 / 22
\end{aligned}
$$

and

$$
\begin{aligned}
& 5 \phi^{2} / 2=(21+\mathrm{k}) /(22+\mathrm{k}) \\
& =(21.18033989) /(22.18033979) \\
& \quad 21 / 22
\end{aligned}
$$

where $\mathrm{k}=\phi^{3}\left(1-\phi^{3}\right)$ is the topological mass of 'tHooft's renormalon which could be thought of as made of two entangleon $\phi^{5}$ particles because $\mathrm{k}=2 \phi^{5}$, our main result follows, namely that

$$
\begin{aligned}
& E=E(O)+E(D) \\
& =m c^{2} / 22+m c^{2}(21 / 22) \\
& =m c^{2} \\
& =E(\text { Einstein }) .
\end{aligned}
$$

So far we have looked at a single monadic building block of our space [63]. However due to self similarity we expect the above relation to hold true on all scales [4-7, 68,69]. To show that this expectation is correct and leads to our fundamental conclusion about the measure concentration, we reason as follows:

Our E-infinity spae is infinite dimensional $n=\infty$, however it has a finite expectation value of $\langle n\rangle=4+\phi^{3}$ and $\left\langle d_{c}\right\rangle=4+\phi^{3}$ for its topological and Hausdorff dimension apart of the Menger-Urysohn topological dimension of exactly $4[68,69]$. Thus although infinite dimensional, because of its hierarchal geometry reflected in its Suslin expansion $[71,76]$

$$
\begin{aligned}
& \operatorname{dim} E-\text { infinity }=\sum_{n=0}^{\infty}\left(\phi^{n}\right)(n) \\
& =(1+\phi) /(1-\phi) \\
& =4+\phi^{3}
\end{aligned}
$$

it is still compact with a boundary at infinity [62-70]. This shows up clearly in the hyperbolic projection of E-infinity space which manifests itself as a modular fractal space of the
Klein-Penrose type $[68,69]$ as explained in great detail in previous older and more recent publications. Now our result follows because in the Poincare-Beltrami projection we have at the center about 336 degrees of freedom corresponding to the internal dimension of the original Klein modular space $[68,69]$

$$
\begin{aligned}
& |\Gamma(7)|=|S L(2,7)| \\
& =7\left(7^{2}-1\right) \\
& =336 .
\end{aligned}
$$

This represents the holographic boundary without the 'compactified' edge. At this edge however, which is located at infinity, we have infinitely more degrees of freedom. Thus although the edge seems to us from the middle to be infinitely thin it is really where the bulk of absolute measure is located, namely the $95.5 \%$. For us low dimensional creatures, the situation may strike us as paradoxical especially when we are making measurements of the total energy density of the cosmos. In this sense the COBE, WMAP and Planck measurement [63-76] makes absolute sense when we realize that this $95.5 \%$ is related to the quantum wave of the universe which collapses on measurement and cannot be detected but can be indirectly inferred from cosmological observations extended to the edge of the accelerated expansion of the universe [4-51].

\section{Validation of the $96 \%$ Result via the Fundamental Renormalization Equation of Electromagnetism}

We recall from E-infinity theory that the reconstruction of the inverse electromagnetic fine structure constant $\bar{\alpha}_{o}=137+k_{o}$ where $k_{o}=\phi^{5}\left(1-\phi^{5}\right)$ and $\phi^{5}=\left(\frac{\sqrt{5}-1}{2}\right)^{5}$ is Hardy's quantum entanglement probability, plays a fundamental role $[68,69]$

$$
\bar{\alpha}_{o}=\left(\bar{\alpha}_{1}\right)(1 / \phi)+\left(\bar{\alpha}_{2}=\bar{\alpha}_{1} / 2\right)+\bar{\alpha}_{3}+\bar{\alpha}_{4}
$$

Here $\bar{\alpha}_{1}=60, \bar{\alpha}_{2}=30, \bar{\alpha}_{3}=9$ and $\bar{\alpha}_{4}=1$ as explained in full detail on many previous occasions $[5,6]$. Consequently one finds the following noteworthy result, namely $[36,50,63]$

$$
\begin{aligned}
& \bar{\alpha}_{o}=\left(97+k_{o}\right)+40 \\
& =137+k_{o}
\end{aligned}
$$

and

$$
\begin{aligned}
& \sum_{i=1}^{4} \bar{\alpha}_{i}=(10)^{2} \\
& =100 .
\end{aligned}
$$


It is easily seen from the above that the normed or normalized infinity of hierarchal dimensions of E-infinity become exactly 100 . These hundred quasi dimensions or degrees of freedom may be interpreted as internal and external isometries. Consequently after taking out the $\mathrm{D}=4$ of Einstein's spacetime and the 22 compactified dimensions of bosonic or strong interaction strings, we are left with 74 isometries which we identify with pure dark energy without the 22 dark matter [10]. In other words the dark section is simply $100-4=96$ which corresponds directly to the $96 \%$ volume concentration discussed earlier on. The reader can easily see that $4 \%, 22 \%$ and $74 \%$ correspond perfectly to the exact values and are merely the integer version of these values obtained not merely by rounding it but from an exact 'renormalized integer' analysis.

It is also a mathematical delight to note that we could proceed in the opposite direction and obtain again in an exact integer analysis the exact integer value of $\bar{\alpha}_{o}$, namely $\bar{\alpha}_{o}$ without the Hardy entanglement tail $k_{o}=\phi^{5}\left(1-\phi^{5}\right)$ which means $\bar{\alpha}_{o}=137$ representing a famous prime number. To do that we need a simple result obtained many years ago when E-infinity was applied to Penrose twistors [78]. There we found that $\phi$ could be written as (1/2) plus a 'boost'. This boost is given by $(1+k) / 10$ where $k=\phi^{3}\left(1-\phi^{3}\right)=2 \phi^{5}$ is 'tHooft's dimensional regularization [4] order parameter $\in=4-D$. It follows then that the following transformation is consistent, namely [78]

$$
1 / \phi=1+\phi \Rightarrow 1+(1 / 2) \text {. }
$$

The corresponding renormalization integer equation, i.e. without any transfinite correction is

$$
\begin{aligned}
& \bar{\alpha}_{o}=[1+(1 / 2)](74)+22+4 \\
& =(74+37)+26 \\
& =137 .
\end{aligned}
$$

In the above equation we have of course inverted the situation which is basically one of the many dualities existing in E-infinity theory as noted in various remarkable papers on E-infinity [7] and Witten's M-theory [10,21,23]. For the benefit of the non-specialist reader we stress that all the above equations are not simply numbers but numbers anchored in well established theories. In addition the $96 \%$ concentration does not stand there alone but is supported by the various other topological and quantum mechanical entanglement arguments developed in the present and previous work [32-44].

\section{Relevance to Holographic Dark Energy [81-83] and the Fundamental Notion of Endophysics $[\mathbf{9 7 , 8 0 ]}$}

Finding with geometrical-topological reasoning that almost $96 \%$ of the volume of certain Banach-like high dimensional manifolds are concentrated near to the surface, i.e. the boundary of the said manifold, naturally invites analogy or even more than analogy to the holographic dark energy [81]. We can thus give a rather accurate estimation of pure dark energy using 'tHooft-Susskin holographic principle [81-83] Based on this principle we can assume following Miao Li's proposal [81] that short distance cut-off is related to the infrared cut-off, then assuming that the infrared cut-off relevant to dark energy is the size of the event horizon one finds the equation of the state of dark energy using the input $\Omega \Lambda=0.73$ [81]. Now this $73 \%$ pure dark energy, i.e. excluding dark matter could be estimated very easily using the isometries of E-infinity theory. In this theory the bulk is given basically by Witten's maximally symmetric manifold $[9,14,21]$

$$
\begin{aligned}
& N_{K}^{(11)}=\left(\begin{array}{c}
11 \\
1
\end{array}\right)+\left(\begin{array}{c}
11 \\
2
\end{array}\right)+\left(\begin{array}{c}
11 \\
5
\end{array}\right) \\
& =11+55+462 \\
& =66+462 \\
& =528 .
\end{aligned}
$$

In turn the corresponding dimension of E8E8 super string space is simply 528 minus the 32 maximal super charge namely [21-24]

$$
\begin{aligned}
& |E 8 E 8|=528-32 \\
& =496 .
\end{aligned}
$$

Thus while the holographic boundary corresponding to 528 requires a five dimensional gravity, that corresponding to 496 is simply the Klein modular curve with its $\operatorname{SL}(2,7)$ symmetry leading to 336 degrees of freedom or isometries because [27-36]

$$
\begin{aligned}
& \operatorname{Dim} \operatorname{SL}(2,7)=7\left(7^{2}-1\right) \\
& =(7)(48) \\
& =336 .
\end{aligned}
$$

From the preceeding well known elementry mathematical facts we can estimate the density of pure dark energy as the ratio of the isometry of the holographic surface plus any coupling to the isometry of the bulk minus any auxillary 'conditional' isometries or coupling. Noting that gravity is the essence of relativity and that we require 528 then for a Kaluza-Klein five dimensional spacetime, we see that the independent Riemannian component, i.e. Riemannian isometries must be $[68,69]$

$$
\begin{aligned}
& R^{(5)}=(5)^{2}\left(5^{2}-1\right) / 12 \\
& =50 .
\end{aligned}
$$

Consequently our pure dark energy density could be estimated by the following ratio [10] 


$$
\begin{aligned}
& \gamma(D) \cong \frac{|S L(2,7)|+R^{(5)}}{N_{K}^{(11)}} \\
& \cong \frac{336+50}{528} \\
& \simeq 0.731 \cong 73 \%
\end{aligned}
$$

which is in fair agreement with the previous estimation of the holographic pure energy. A second upper bound estimate could be found using E8E8 by involving the logarithmic scaling of the ratio of grand unification 'tHooft-Polyakov monopole to the mass of the electroweak $\mathrm{Z}$ particle. This leads to a value equal to the maximal central charge [47]

$$
\begin{aligned}
& C=\ln \frac{M_{u}}{m_{Z}} \\
& =\ln \left(10^{16} / 91\right) \\
& =32 .
\end{aligned}
$$

Consequently one finds from inserting in equation (18) again that [62-69]

$$
\begin{aligned}
& \gamma(D)=\frac{336+32}{496} \\
& \simeq 0.74 \\
& =74 \% .
\end{aligned}
$$

Next we need to address a fundamental question and in fact a superficially fundamental contradiction, namely why all previous experimental test of $\mathrm{E}=\mathrm{mc}^{2}$ did not find any derivation amounting to anything even remotely near to the $96 \%$ or in the pure case $73 \%$ missing dark energy? The clue to this riddle could be found in the global nature of any $\mathrm{E}=\mathrm{mc}^{2}$ experiment. The situation is analogous and in fact deeply related to the phenomenon of quantum wave interference. This interference is absent in any endophysical experiment. Similarly $\mathrm{E}=\mathrm{mc}^{2} / 22$ is exophysically absent in any experiment. Thus to observe $\mathrm{E}=\mathrm{mc}^{2} / 22+\mathrm{mc}^{2}(21 / 22)$ we need to perform an endophysical measurement. None of the many tests referred to in the literature could be described as endophysical. By contrast the COBE, WMAP as well as Plank and type 1a supernova analysis can safely be described as a true endophysical experiment $[79,80]$. In other words the existence or rather absece of dark energy is the proof that Einstein's famous formula is correct and that it is only naturally blind to the distinction between the two components contained in it. The situation is most vividly illustrated by the skiing observer looking upon another skier in fractal land via the fractal-non fractal interface (see Fig. 1.) The figure is only scantily related or inspired by James Joyce's novel "A portrait of the artist as a young man".

\section{Discussion}

The quantum wave and wave collapse may be a familiar face of the quantum world but this does not change its deep status as the greatest puzzle in phyics. How could something which has no energy, no matter and no momentum have such a crutial effect on the motion of quantum objects. Suddenly however cosmology started becoming equally puzzling, if not more puzzling, with the discovery of a type of energy which may be the possible cause behind the accelerated cosmic expansion of the universe athough it is de facto not there because it cannot be measured and if we try very hard, we find only $4.5 \%$ ordinary energy and ordinary matter leaving the $95.5 \%$ shrouded in mystery. These things which are there but are really not there remind anyone working in deterministic chaos, nonlinear dynamics and fractals with the paradoxical nature of a Cantor set $[68,69]$. These transfinite sets possess no measure, being of measure zero which means no length at all and consequently 'physically' not there. However they do have a substantial Hausdorff dimension, namely $\ln 2 / \ln 3 \simeq 0.63$ for the original Cantor set and $\phi=0.618033989$ for a uniformally random Mauldin-Williams Cantor set. Now we ask ourselves how did we succeed in applying an Occam razon magnum to these three paradoxical factors? The short answer is by reducing dark energy to the non-ordinary energy of the quantum wave and the quantum wave to an empty set dimension $\phi^{2}$ which happens to be the surface of the zero set quantum particle with a Hausdorff dimension $\phi$. That way we seem to have eliminated the problem by illuminating it via the light of fractal Cantorian geometry and transfinite set theory as well as the associated E-infinity algebra of the highly structured golden mean ring which can deal with the most complex computation with unheard of simplicity $[68,69,76]$. From this view point dark energy could be detected only via an endophysical experiment such as COBE, WMAP and type 1a supernova. In other words $E=m c^{2} / 22$ and $E=m c^{2}(21 / 22)$ dark energy are theoretical and experimental confirmation of Einstein's theory and not a refutation. We may also add that the present measure concentration harmonizes perfectly with J.A. Wheeler's famous principle of the boundary of a boundary is equal zero and we may interpret the edge of the holographic boundary of our universe as a one sided Möbius strip bringing Casimir energy and dark energy together.

\section{Conclusion}

The present work clearly shows that the disection of Einstein's energy density into two parts is a completely natural consequence of a very high dimensional geometry and its associated measure concentration which results in almost $96 \%$ of the volume being located near to the surface of the concerned manifold $[58,61]$. The same conclusion is reached via Hardy's quantum entanglement $\mathrm{P}=\phi^{5}$ [5]. Applied to our cosmos as modelled by E-infinity Cantorian spacetime manifold, the missing endophysical dark energy of the Hartle-Hawking wave function of the universe becomes a natural consequence of the geometry and topology of E-infinity as confirmed by the endophysical measurement of WMAP and type 1a supernova. Together with our anticlastic, anti-curvature and antigravity explanation of real material-like spacetime [26], dark energy and accelerated cosmic expansion find in the above a rational mathematical-geometrical and 
physical explanation which harmonizes with the holographic principle [81-83] and supports the conclusion of numerous other theories which consider dark energy to be the negative gravitational force behind the observed accelerated cosmic expansion. Finally the main result, namely the $96 \%$ Banach-like manifold volume concentration is validated based on the electromagnetic renormalization equations of E-infinity theory. Most of the present conclusions are summarized metaphorically in the landscape of Fig. 1.

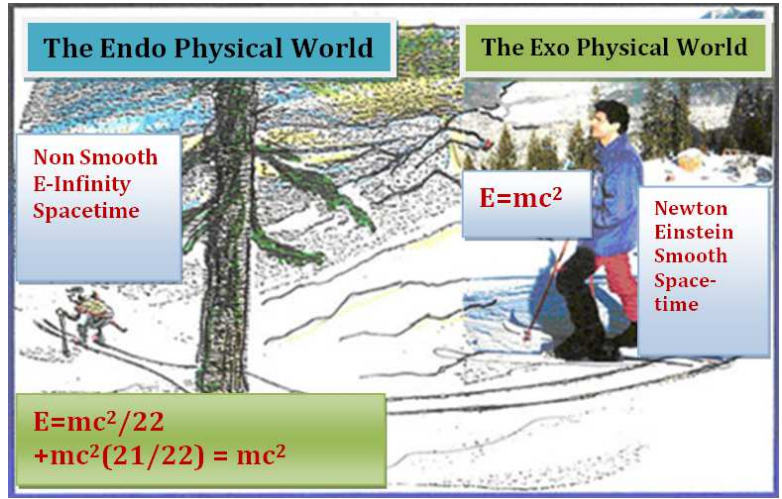

Fig. 1. A skier in the classical exophysical world observing via the "interface" another fractal skier in a fractal universe performing impossible tricks without really being astonished. The fractal endophysical universe is really infinite dimensional and there is no problem in going around the tress in two ways simultaneously without smashing into the tree. In the classical world we are performing exophysical experiments where $E=m c^{2}$. In the fractal universe however we are in an endophysical universe and $E$ is split in $E_{1}=$ $m c^{2} / 22$ plus $E_{2}=m c^{2}(21 / 22)$ where $E_{1}+E_{2}=m c^{2}$, confirming rather than contradicting Einstein's formula. Again it is the high dimensionality of a Banach space which does the trick in a fractal E-infinity space. This is a direct illustration of the importance of the topological invariant we call 'dimensionality' in real objects such as the two dimensional Möbius strip [16] and the three dimensional Klein bottle where there is no 'inside' and 'outside'. Here the two ways of the skier are only an illusion and we have only one way. This is possible and similar to a Banach space [58-61].

\section{References}

[1] W. Rindler, Relativity (Special, General and Cosmological). Oxford University Press, Oxford. 2004.

[2] L.B. Okun, Energy and mass in relativity theory. World Scientific, Singapore 2009.

[3] W. Rindler, Introduction to Special Relativity. Oxford Science Publications, Oxford 1991.

[4] Mohamed S. El Naschie, On a New Elementary Particle from the Disintegration of the Symplectic 'tHooft-Veltman-Wilson Fractal Spacetime. World Journal of Nuclear Science and Technology, 4(4), 2014, pp. 216-221.

[5] M. A. Helal, L. Marek-Crnjac, Ji-Huan He, The Three Page Guide to the Most Important Results of M. S. El Naschie's Research in E-Infinity Quantum Physics and Cosmology. Open Journal of Microphysics, 3(4), 2013, pp. 141-145.

[6] L. Marek-Crnjac, Ji-Huan He, An Invitation to El Naschie's Theory of Cantorian Space-Time and Dark Energy. International Journal of Astronomy and Astrophysics, 3(4), 2013, pp. 464-471.
[7] Jean-Paul Auffray, E-Infinity Dualities, Discontinuous Spacetimes, Xonic Quantum Physics and the Decisive Experiment. Journal of Modern Physics, 5(15), 2014, pp. $1427-1436$.

[8] Mohamed S. El Naschie, Quantum Entanglement as a Consequence of a Cantorian Micro Spacetime Geometry. Journal of Quantum Information Science, 1(2), 2011, pp. 50-53.

[9] Mohamed S. El Naschie, A Resolution of Cosmic Dark Energy via a Quantum Entanglement Relativity Theory. Journal of Quantum Information Science, 3(1), 2013, pp. 23-26.

[10] M. S. El Naschie, A Unified Newtonian-Relativistic Quantum Resolution of the Supposedly Missing Dark Energy of the Cosmos and the Constancy of the Speed of Light. International Journal of Modern Nonlinear Theory and Application, 2(1), 2013, pp. 43-54.

[11] Mohamed S. El Naschie, Quantum Entanglement: Where Dark Energy and Negative Gravity plus Accelerated Expansion of the Universe Comes From. Journal of Quantum Information Science, 3(2), 2013, pp. 57-77.

[12] Mohamed S. El Naschie, A Fractal Menger Sponge Space-Time Proposal to Reconcile Measurements and Theoretical Predictions of Cosmic Dark Energy. International Journal of Modern Nonlinear Theory and Application, 2(2), 2013, pp. 107-121.

[13] Mohamed S. El Naschie, The Hydrogen Atom Fractal Spectra, the Missing Dark Energy of the Cosmos and Their Hardy Quantum Entanglement. International Journal of Modern Nonlinear Theory and Application, 2(3), 2013, pp. 167-169.

[14] Mohamed S. El Naschie, A Rindler-KAM Spacetime Geometry and Scaling the Planck Scale Solves Quantum Relativity and Explains Dark Energy. International Journal of Astronomy and Astrophysics, 3(4), 2013, pp. 483-493.

[15] M. S. El Naschie, What Is the Missing Dark Energy in a Nutshell and the Hawking-Hartle Quantum Wave Collapse. International Journal of Astronomy and Astrophysics, 3(3), 2013, pp. 205-211.

[16] Mohamed S. El Naschie, The Missing Dark Energy of the Cosmos from Light Cone Topological Velocity and Scaling of the Planck Scale. Open Journal of Microphysics, 3(3), 2013, pp. 64-70.

[17] Mohamed S. El Naschie, From Yang-Mills Photon in Curved Spacetime to Dark Energy Density. Journal of Quantum Information Science, 3(4), 2013, pp. 121-126.

[18] Mohamed S. El Naschie, Calculating the Exact Experimental Density of the Dark Energy in the Cosmos Assuming a Fractal Speed of Light. International Journal of Modern Nonlinear Theory and Application, 3(1), 2014, pp. 1-5.

[19] Mohamed S. El Naschie, Cosmic Dark Energy Density from Classical Mechanics and Seemingly Redundant Riemannian Finitely Many Tensor Components of Einstein's General Relativity. World Journal of Mechanics, 4(6), 2014, pp. 153-156.

[20] Mohamed S. El Naschie, Capillary Surface Energy Elucidation of the Cosmic Dark Energy-Ordinary Energy Duality. Open Journal of Fluid Dynamics, 4(1), 2014, pp. 15-17. 
[21] Mohamed S. El Naschie, Einstein's General Relativity and Pure Gravity in a Cosserat and De Sitter-Witten Spacetime Setting as the Explanation of Dark Energy and Cosmic Accelerated Expansion. International Journal of Astronomy and Astrophysics, 4(2), 2014, pp. 332-339.

[22] Mohamed S. El Naschie, Electromagnetic-Pure Gravity Connection via Hardy's Quantum Entanglement. Journal of Electromagnetic Analysis and Applications, 6(9), 2014, pp. 233-237.

[23] Mohamed S. El Naschie, Cosmic Dark Energy from 't Hooft's Dimensional Regularization and Witten's Topological Quantum Field Pure Gravity. Journal of Quantum Information Science, 4(2), 2014, pp. 83-91.

[24] Mohamed S. El Naschie, Entanglement of E8E8 Exceptional Lie Symmetry Group Dark Energy, Einstein's Maximal Total Energy and the Hartle-Hawking No Boundary Proposal as the Explanation for Dark Energy. World Journal of Condensed Matter Physics, 4(2), 2014, pp. 74-77.

[25] Mohamed S. El Naschie, The Meta Energy of Dark Energy. Open Journal of Philosophy. 4(2), 2014, pp. 157-159.

[26] Mohamed S. El Naschie, Pinched Material Einstein Space-Time Produces Accelerated Cosmic Expansion. International Journal of Astronomy and Astrophysics. 4(1), 2014, pp. 80-90.

[27] Mohamed S. El Naschie, From Chern-Simon, Holography and Scale Relativity to Dark Energy. Journal of Applied Mathematics and Physics, 2(7), 2014, pp. 634-638.

[28] Mohamed S. El Naschie, Why $E$ Is Not Equal to $m c^{2}$. Journal of Modern Physics, 5(9), 2014, pp. 743-750.

[29] Mohamed S. El Naschie, Nash Embedding of Witten's M-Theory and the Hawking-Hartle Quantum Wave of Dark Energy. Journal of Modern Physics, 4(10), 2013, pp. 1417-1428.

[30] Mohamed S. El Naschie, Dark Energy from Kaluza-Klein Spacetime and Noether's Theorem via Lagrangian Multiplier Method. Journal of Modern Physics, 4(6), 2013, pp. 757-760.

[31] Mohamed S. El Naschie, The hyperbolic Extension of Sigalotti-Hendi-Sharifzadeh's Golden Triangle of Special Theory of Relativity and the Nature of Dark Energy. Journal of Modern Physics, 4(3), 2013, pp. 354-356.

[32] Mohamed S. El Naschie, Topological-Geometrical and Physical Interpretation of the Dark Energy of the Cosmos as a 'Halo' Energy of the Schrodinger Quantum Wave. Journal of Modern Physics, 4(5), 2013, pp. 591-596.

[33] M. S. El Naschie, From Modified Newtonian Gravity to Dark Energy via Quantum Entanglement. Journal of Applied Mathematics and Physics, 2(8), 2014, pp. 803-806.

[34] Ji-Huan He, L. Marek-Crnjac, Mohamed El Naschie's Revision of Albert Einstein's $E=m_{0} c^{2}$ : A Definite Resolution of the Mystery of the Missing Dark Energy of the Cosmos International. Journal of Modern Nonlinear Theory and Application, 2(1), 2013, pp. 55-59.

[35] M. S. El Naschie, L. Marek-Crnjac, Deriving the Exact Percentage of Dark Energy Using a Transfinite Version of Nottale's Scale Relativity. International Journal of Modern Nonlinear Theory and Application, 1(4), 2012, pp. 118-124.
[36] Mohamed S. El Naschie, Atef Helal, Dark Energy Explained via the Hawking-Hartle Quantum Wave and the Topology of Cosmic Crystallography. International Journal of Astronomy and Astrophysics, 3(3), 2013, pp. 318-343.

[37] L. Marek-Crnjac, Mohamed S. El Naschie: Chaotic Fractal Tiling for the Missing Dark Energy and Veneziano Model. Applied Mathematics, 4(11B), 2013, pp. 22-29.

[38] L. Marek-Crnjac, M. S. El Naschie, Quantum Gravity and Dark Energy Using Fractal Planck Scaling. Journal of Modern Physics, 4(11A), 2013, pp. 31-38.

[39] L. Marek-Crnjac, Mohamed S. El Naschie, Ji-Huan He, Chaotic Fractals at the Root of Relativistic Quantum Physics and Cosmology. International Journal of Modern Nonlinear Theory and Application, 2(1A), 2013, pp. 78-88.

[40] M. S. El Naschie, S. Olsen, J. H. He, S. Nada, L. Marek-Crnjac, A. Helal, On the Need for Fractal Logic in High Energy Quantum Physics. International Journal of Modern Nonlinear Theory and Application, 1(3), 2012, pp. 84-92.

[41] Mohamed Salah El Naschie, Leila Marek-Crnjac, Mohamed Atef Helal, Ji-Huan He, A Topological Magueijo-Smolin Varying Speed of Light Theory, the Accelerated Cosmic Expansion and the Dark Energy of Pure Gravity. Applied Mathematics, 5(12), 2014, pp. 1780-1790.

[42] Mohamed S. El Naschie, Compactified dimensions as produced by quantum entanglement, the four dimensionality of Einstein's smooth spacetime and 'tHooft's 4- $\mathcal{E}$ fractal spacetime. American Journal of Astronomy \& Astrophysics, 2(3), 2014, pp. 34-37.

[43] Mohamed S. El Naschie, Hardy's Entanglement as the Ultimate Explanation for the Observed Cosmic Dark Energy and Accelerated Expansion. International Journal High Energy Physics, 1(2), 2014, pp. 13-17.

[44] Mohamed S. El Naschie, Deriving E $=\mathrm{mc}^{2} / 22$ of Einstein's ordinary quantum relativity energy density from the Lie symmetry group $\mathrm{SO}(10)$ of grand unification of all fundamental forces and without quantum mechanics. American Journal of Mechanics \& Applications, 2(2), 2014, pp. 6-9.

[45] Mohamed S. El Naschie, Cosserat-Cartan Modification of Einstein-Riemann Relativity and Cosmic Dark Energy Density. American Journal of Modern Physics. 3(2), 2014, pp. 82-87.

[46] Mohamed S. El Naschie, Asymptotically safe pure gravity as the source of dark energy of the vacuum. Int. Journal Astrophysics \& Space Science, 2(1), 2014, pp. 12-15.

[47] Mohamed S. El Naschie, Logarithmic Running of't Hooft-Polyakov Monopole to Dark Energy. International Journal of High Energy Physics, 1(1), 2014, pp. 1-5.

[48] Mohamed S. El Naschie, Experimentally Based Theoretical Arguments that Unruh's Temperature, Hawking's Vacuum Fluctuation and Rindler's Wedge Are Physically Real. American Journal of Modern Physics, 2(6), 2013, pp. 357-361.

[49] L. Marek-Crnjac, Modification of Einstein's $E=\mathrm{mc}^{2}$ to $\mathrm{E}=$ $(1 / 22) \mathrm{mc}^{2}$. American Journal of Modern Physics, 2(5), 2013, pp. 255-263.

[50] M.S. El Naschie, The quantum gravity Immirzi parameter - A general physical and topological interpretation. Gravitation and Cosmology, 19(3), 2013, pp. 151-155. 
[51] M.S. El Naschie, Determining the missing dark energy density of the cosmos from a light cone exact relativistic analysis. Journal of Physics, 2(2), 2013, pp. 19-25.

[52] M.S. El Naschie, The quantum entanglement behind the missing dark energy. Journal of Modern Physics and Applications, 2(1), 2013, pp. 88-96.

[53] M.S. El Naschie, Dark energy via quantum field theory in curved spacetime. Journal Modern Physics and Applications, 2, 2014, pp. 1-7.

[54] M.S. El Naschie, Rindler space derivation of dark energy. Journal of Modern Physics Applications. 6, 2014, pp. 1-10.

[55] Wei Tang et al, From nonlocal elasticity to nonlocal spacetime and nano science. Bubbfil Nanotechnology. 1(1), 2014, pp. $3-12$.

[56] M.S. El Naschie, To dark energy theory from a Cosserat-like model of spacetime. Problems of Nonlinear Analysis in Engineering Systems. 1(41), Vol. 20, 2014, pp. 79-98.

[57] M.S. El Naschie, Revising Einstein's E $=\mathrm{mc}^{2}$; A theoretical resolution of the mystery of dark energy. Proceedings of the Fourth Arab Int. Conference in Physics and Material Science, Egypt. 1-30 October, 2012, pp. 1.

[58] K.M. Ball: Volume ratios and a reverse isoperimetric inequality. Journal of London Mathematical Society. 44, 1991, pp. 351-359.

[59] G. Pisier, The volume of convex bodies and Banach space geometry. Tracts in Math 94, Cambridge University Press, Cambridge, 1989.

[60] B.S. Kasin, The width of certain finite-dimensional sets and classes of smooth functions. IZV. Akad. Nauk. SSSR. Ser. Mat. 41(2), 1977, pp. 334-351 (in Russian).

[61] O. Guedon, Concentration phenomena in high dimensional geometry. arXiv:1310.1204V1[math]4 Oct 2013.

[62] Ji-Huan He (Guest Editor), Fractal Spacetime and Noncommutative Geometry in Quantum and High Energy Physics. 3(1). Special issue on recent developments on dark energy and dark matter, 2013, pp. 1-62.

[63] Ji-Huan He, M.S. El Naschie, On the monadic nature of quantum gravity as a highly structured golden ring, spaces and spectra. Fractal Spacetime and Noncommutative Geometry in Quantum and High Energy Physics. 2(2), 2012, pp. 94-98.

[64] M.S. El Naschie, Towards a general transfinite set theory for quantum mechanics. Fractal Spacetime and Noncommutative Geometry in Quantum and High Energy Physics. 2(2), 2012, pp. 135-142.

[65] M.S. El Naschie, Ji-Huan He, S. Nada, L. Marek-Crnjac, M. Helal, Golden mean computer for high energy physics. Fractal Spacetime and Noncommutative Geometry in Quantum and High Energy Physics. 2(2), 2012, pp. 80-92.

[66] M.S. El Naschie, The minus one connection of relativity, quantum mechanics and set theory. Fractal Spacetime and Noncommutative Geometry in Quantum and High Energy Physics. 2(2), 2012, pp. 131-134.

[67] M.S. El Naschie, Dark energy and its cosmic density from Einstein's relativity and gauge fields renormalization leading to the possibility of a new 'tHooft quasi particle. The Open Journal of Astronomy. In press.
[68] M.S. El Naschie, A review of E-infinity and the mass spectrum of high energy particle physics. Chaos, Solitons \& Fractals, 19(1), 2004, pp. 209-236.

[69] M.S. El Naschie, The theory of Cantorian spacetime and high energy particle physics (An informal review). Chaos, Solitons \& Fractals, 41, 2009, pp. 2635-2646.

[70] A. Connes, Noncommutative Geometry. Academic Press, San Diego, USA, 1994.

[71] S.G. Krantz and H.R. Parks, Geometric Integration Theory. Birkhauser, Boston, USA, 2008.

[72] M.S. El Naschie, Remarks on super strings, fractal gravity, Nagasawa's diffusion and Cantorian spacetime. Chaos, Solitons \& Fractals, 8(11), 1997, pp. 1873-1886.

[73] M.S. El Naschie, Introduction to nonlinear dynamics, general relativity and the quantum - The uneven flow of fractal time. Chaos, Solitons \& Fractals, 8(5), 1997, pp. vii-x.

[74] M.S. El Naschie, Hyper-dimensional geometry and the nature of physical spacetime. Chaos, Solitons \& Fractals, 10(1), 1999, pp. 155-158.

[75] M.S. El Naschie, E-infinity - High Energy Communications Nos. 1-90. April 2010 to December 2012.

[76] Chen Nanxian, Möbius inversion in physics. World Scientific, Singapore 2010.

[77] Ji-Huan He, A tutorial review on fractal spacetime and fractional calculus. Int. Journal of Theoretical Physics, 53(11), 2014, pp. 3698-3718.

[78] M.S. El Naschie, On twistors in Cantorian E-infinity space. Chaos, Solitons \& Fractals, 12(4), 2001, pp. 741-746.

[79] O.E. Rössler, Endophysics, World Scientific, Singapore (1998).

[80] M.S. El Naschie, On a general theory for quantum gravity. In 'Science of the Interface. Editor H. Diebner, T. Druckrey and P. Weibel. Genista Verlag, Tübingen, Germany, 2001.

[81] Miao Li, A model of holographic dark energy. Physics Letters B, 603(1-2), 2004, pp. 1-5.

[82] M.S. El Naschie, Holographic dimensional reduction. Center manifold theorem and E-infinity. Chaos, Solitons \& Fractals, 29(4), 2006, pp. 816-822.

[83] A.P. Balachandran, S. Kürkcüoglu, S. Vaidya, Lectures on fuzzy and fuzzy Susy physics. World Scientific, Singapore 2007.

[84] J. Bahcall, T. Pivan and S. Weinberg, Dark Matter in The Universe. Second Edition. World Scientific, Singapore, 2004.

[85] L. Amendola and S. Tsujikawa, Dark Energy - Theory and Observation. Cambridge University Press, Cambridge, 2010.

[86] P. Ruiz-Lapuente, Dark Energy, Observational and Theoretical Approaches. Cambridge University Press, Cambridge, 2010.

[87] M.S. El Naschie, From $\mathrm{E}=\mathrm{mc}^{2}$ to $\mathrm{E}=\mathrm{mc}^{2} / 22-\mathrm{A}$ short account of the most famous equation in physics and its hidden quantum entangled origin. Journal of Quantum Information Science, 4, 2014, pp. 284-291. 
[88] M.S. El Naschie, Casimir-like energy as a double Eigenvalue of quantumly entangled system leading to the missing dark energy density of the cosmos. Intnational Journal of High Energy Physics, 1(5), 2014, pp. 55-63.
[89] S. Perlmutter et al, Supernova cosmology project collaboration "Measurements of omega and lambda from 42 high redshift supernova. Astrophysics Journal, 517, 1999, pp. 565-585. 